\title{
Associação de variáveis ambientais à ocorrência de leptospirose canina e humana na cidade de São Paulo
}

\author{
[Association of environmental variables with the leptospirosis occurrence in dogs and \\ humans at São Paulo city] \\ R.I. Cipullo, R.A. Dias ${ }^{*}$ \\ Faculdade de Medicina Veterinária e Zootecnia - USP - São Paulo, SP
}

\begin{abstract}
RESUMO
Foi realizada uma análise espacial da ocorrência de leptospirose humana e canina na Supervisão de Vigilância em Saúde do Butantã, situada no município de São Paulo, no ano de 2007, associada a variáveis ambientais de risco, tais como: focos de enchente e áreas de desratização. Foram encontrados aglomerados espaciais de pontos de alagamentos em 12 setores censitários e de casos de leptospirose humana em quatro setores censitários, sem correlação entre ambos. Não foram encontrados agrupamentos de casos em cães, possivelmente devido à subnotificação. As proporções casos humanos de leptospirose : população humana dentro e fora da área de desratização foram 7:199.600 e 9:257.980, respectivamente. Conclui-se que medidas de controle de roedores como a desratização foram responsáveis pela minimização dos efeitos dos fatores de risco para a transmissão de leptospirose para humanos.
\end{abstract}

Palavras-chave: Leptospira sp., antropozoonose, estatística espacial, sistema de informação geográfica, fatores de risco ambientais

\begin{abstract}
A spatial analysis of the human and canine leptospirosis occurrence was performed in São Paulo city in 2007, associated with environmental risk variables such as flooding and rodent control sites. Clusters of flooding sites were found in 12 census sectors, and human leptospirosis in 4 census sectors, without correlation between them. Clusters of canine cases were not found, possibly due to lack of notification. The proportions of human cases in and out of rodent control areas were, respectively, 7:199,600 and 9:257,980. Rodent control measures minimized the effects of the risk factors in the leptospirosis transmission to humans.
\end{abstract}

Keywords: antropozoonosis, Leptospira sp., spatial statistics, geographic information systems, environmental risk factors

\section{INTRODUÇÃ̃o}

A leptospirose é uma das doenças de caráter antropozoonótico mais difundida no mundo (OIE, 2006). Sua incidência aumenta quando ocorre maior precipitação pluviométrica (Barcellos et al., 2001), variando de acordo com cada região no Brasil. Na cidade de São PauloSP, há um agravante na questão da ocorrência de leptospirose: a constante inundação de áreas urbanas e moradias (FUNASA, 1997). A região

Recebido em 27 de abril de 2011

Aceito em 31 de janeiro de 2012

*Autor para correspondência (corresponding author)

E-mail: dias@vps.fmvz.usp.br
Centro-Oeste da cidade de São Paulo é composta por quatro Supervisões de Vigilância em Saúde SUVIS: Butantã, Pinheiros, Lapa e Sé, localizadas nas áreas de maiores incidências de leptospirose humana entre 1998 e 2006 (Soares et al., 2010). A área da administração da SUVIS do Butantã, em especial, realizava o georreferenciamento das ações relacionadas ao controle de roedores, como a desratização.

Um dos fatores que pode influenciar a incidência de leptospirose em uma região é a ocupação de 
áreas irregulares com alta ocorrência de enchentes e falta de saneamento e de coleta de lixo, o que propicia a infestação por roedores (De Paula, 2005). Outro fator de grande importância para a disseminação da leptospirose é a quantidade de infectados presentes na região, pois a infecção pode ocorrer pelo contato com urina, água e solo contaminados com leptospiras viáveis (de Almeida et al., 1994; Ibarra et al., 2003).

Os principais responsáveis pela contaminação do ambiente urbano por leptospiras são os roedores, pois atuam como reservatórios de leptospiras e não apresentam sinais clínicos de infecção, porém eliminam as leptospiras durante vários dias e meses (Acha e Szyfres, 2003). As espécies urbanas de roedores mais importantes nesse contexto são: Rattus rattus e Rattus norvegicus.

Os objetivos deste trabalho foram extrair o máximo de informações a respeito da epidemiologia da doença em humanos e em cães da região da SUVIS Butantã e salientar a importância de fatores ambientais na ocorrência da leptospirose.

\section{MATERIAL E MÉTODOS}

Foram obtidos, junto ao Instituto Brasileiro de Geografia e Estatística (IBGE 2002), os seguintes mapas georreferenciados da SUVIS Butantã: distritos administrativos - Rio Pequeno, Raposo Tavares, Butantã, Vila Sônia e Morumbi - e seus respectivos setores censitários, rede hidrográfica, base das áreas de favelas de São Paulo e população humana por setores censitários em 2002.

Junto à SUVIS do Butantã, foram obtidas as seguintes informações: localização dos casos de leptospirose humana ocorridos em 2007, localização das áreas de atenção do programa de controle de roedores - que se encontram próximo a córregos, favelas e regiões carentes de saneamento básico - abrangência geográfica de ação dos produtos utilizados para a desratização - granulado, bloco parafinado e pó de contato no programa de controle.

O Hospital Veterinário (HOVET) da Faculdade de Medicina Veterinária e Zootecnia da USP disponibilizou informações sobre data do diagnóstico e localização da residência dos cães infectados em 2007.

O banco de dados referente aos alagamentos ocorridos em vias públicas na região da SUVIS Butantã, no ano de 2007, foi obtido na página da internet do Centro de Gerenciamento de Emergências (CGE, 2008).

Foi utilizada a localização dos casos de leptospirose canina atendidos no HOVET em 2007, juntamente com a informação sobre a população canina, que, neste caso, foi baseada hipoteticamente na razão homem:cão de 2:1, sendo este dado considerado constante para toda a SUVIS Butantã.

Essas informações foram analisadas no programa Terraview ${ }^{\circledR 3}$.2.1, utilizando-se o método Spatial Scan, que testa a hipótese de os indivíduos possuírem uma mesma probabilidade de se tornarem um caso, ou a hipótese da existência de uma área onde a probabilidade de ser um caso é maior, identificando possíveis aglomerados de casos de cães, em função da população existente.

Os casos de leptospirose humana ocorridos na SUVIS Butantã no período de 2007 foram localizados pelo endereço em um mapa georreferenciado, juntamente com a população humana agregada disposta por setor censitário. Por meio, novamente, do método Spatial Scan, foi feita a análise espacial para verificar e, principalmente, visualizar possíveis aglomerados de casos humanos.

Os fatores de risco para leptospirose humana são conhecidos e, com base na literatura (Acha e Szyfres, 2003), foram identificados como: contato com roedores e presença de corpos d'água, áreas alagáveis e abundância de roedores. Esses fatores são evidentes em áreas sob a atenção do programa de controle de roedores da SUVIS Butantã e também em áreas de ocupação de favelas.

Utilizando-se a informação de que a abrangência geográfica da ação dos produtos usados na desratização é de 50 metros (comunicação pessoal, Di Cezare, 2008 - Supervisão de Vigilância em Saúde do Butantã), a área de desratização foi elaborada traçando-se um raio de 50 metros a partir das ruas que compõem o 
programa de controle de roedores da SUVIS Butantã.

Por meio do script Geoprocessing Wizard 9.2 do ArcGIS® (ESRI INC.), foi recortada a área de desratização do mapa completo e, depois, utilizando-se o script "Area Tools", foi calculada a área dos setores censitários contidos na área de desratização. Uma proporção $\mathrm{P}$ foi gerada a partir das áreas dos setores censitários recortados sobre as áreas dos respectivos setores censitários inteiros. Pressupondo-se que a distribuição populacional é isotrópica dentro deles, foi possível calcular a população de dentro da área de desratização, multiplicando a proporção $\mathrm{P}$ pela população total do respectivo setor censitário. Esse dado é extremamente necessário para comparar as proporções: (a) casos humanos dentro da área de desratização sobre população humana dentro da mesma área e (b) casos humanos fora da área de desratização sobre a população humana na mesma área.

Utilizando-se o banco de dados de alagamentos das vias públicas da SUVIS Butantã no ano de 2007, o programa TerraView ${ }^{\circledR}$ e o método Grimson, analisou-se a existência de aglomerados espaciais. Esse método foi o escolhido, pois necessita apenas dos objetos a serem analisados (casos de alagamentos), ou seja, não faz pressuposição para população de referência.

\section{RESULTADOS E DISCUSSÃO}

As proporções entre casos humanos de leptospirose: população humana dentro e fora da área de desratização foram de 7:199.600 e 9:257.980, respectivamente. O resultado indica que não houve diferença nas duas situações $(\mathrm{P}=0,29)$.

O resultado para a análise Spatial Scan quanto aos casos humanos foi de quatro setores censitários contendo aglomerados de casos $(\mathrm{P}=$ 0,01). Quanto aos casos de cães, não foram identificados aglomerados $(\mathrm{P}=0,21)$. Para o teste de Grimson na análise dos pontos de alagamento, o resultado foi a existência de aglomerados espaciais em 12 setores censitários $(\mathrm{P}<0,001)$.

A leptospirose pode ser separada em duas categorias distintas quanto às implicações clínicas: uma quando o hospedeiro é acometido por um sorovar ao qual ele é adaptado, tornandose, assim, reservatório, a outra quando o hospedeiro não é adaptado ao sorovar, infecção incidental, forma de ocorrência em humanos (Karande et al., 2002). Principalmente na primeira situação, os animais eliminam as leptospiras pela urina por um período de semanas a meses, tornando-se uma fonte de infecção relevante para o homem.

A importância do cão como fonte de infecção de leptospirose para o humano já foi discutida há muito por outros autores (Alves et al., 2000; Brod et al., 2005), porém pouco foi elucidado sobre o real risco do cão infectado na transmissão da leptospirose ao humano nessa região do município de São Paulo. Como há subnotificação dos casos de leptospirose canina, em razão da população de cães errantes e por não haver um sistema de notificação de agravos em cães, os dados sobre leptospirose canina carecem de mais informações.

Devido ao fato de haver acesso apenas aos dados de atendimento de cães com leptospirose do HOVET, não foi possível dizer se há predisposição da transmissão do cão para o humano, pois os casos localizavam-se apenas nas proximidades da USP e não cobriam a totalidade da região da SUVIS Butantã (Fig. 1).

Sabe-se que os fatores de risco para a infecção leptospírica no humano - presença de roedores e corpos d'água - são os mesmos que atuam nos cães (Jouglard et al., 2000) e, portanto, se existissem os dados completos sobre leptospirose canina, a área dos aglomerados de casos de cães provavelmente estaria sobreposta à área dos aglomerados de casos humanos. 


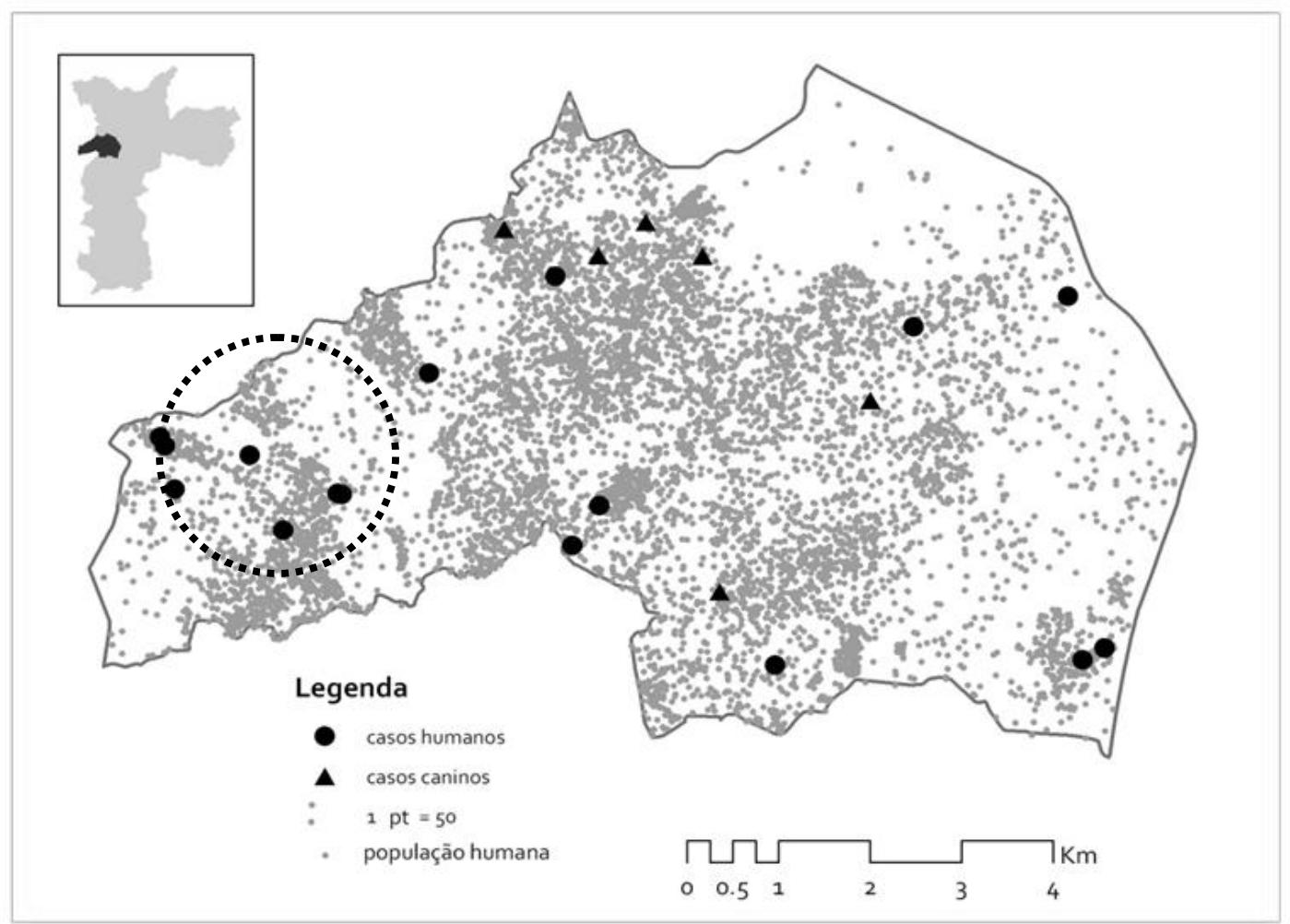

Figura 1. Casos humanos e caninos de leptospirose sobrepostos com a densidade populacional humana na Supervisão de Vigilância em Saúde Butantã em 2007. A área destacada corresponde ao aglomerado de casos humanos.

A importância das áreas de desratização na ocorrência de leptospirose humana é grande (Fig. 2 ), pois era de se esperar que o número de casos fosse mais elevado em seu interior, em razão da grande densidade populacional existente (Fig. 1) e dos fatores de risco preponderantes nessa área: córregos-corpos d'água e área ocupada por favela (Fig. 3). Porém, constatou-se que não houve diferença na ocorrência da doença dentro e fora da área de desratização. Sendo assim, o resultado do teste de duas proporções indicou que o trabalho realizado pela SUVIS Butantã, em controlar o número de roedores por meio de raticidas deixados em bueiros e córregos, auxiliou na diminuição da ocorrência de casos de leptospirose humana nessa região. Com a redução do número de roedores nessa região, que sabidamente possui grande potencial em relação à disseminação da doença, a probabilidade da transmissão roedor-humano também se reduz. Portanto, é admissível afirmar que, se as ações de controle de roedores não fossem executadas nessa área de desratização, a ocorrência da leptospirose seria mais alta que a constada.

O agrupamento de casos humanos, mostrado nas Fig. 1 e 2 pela área destacada, pode ter origem na somatória dos seguintes fatores de risco: alta densidade populacional, presença de grande quantidade de corpos d'água e desratização parcial, que não cobriu completamente a área destacada; isso reforça a importância da desratização no controle da leptospirose. 


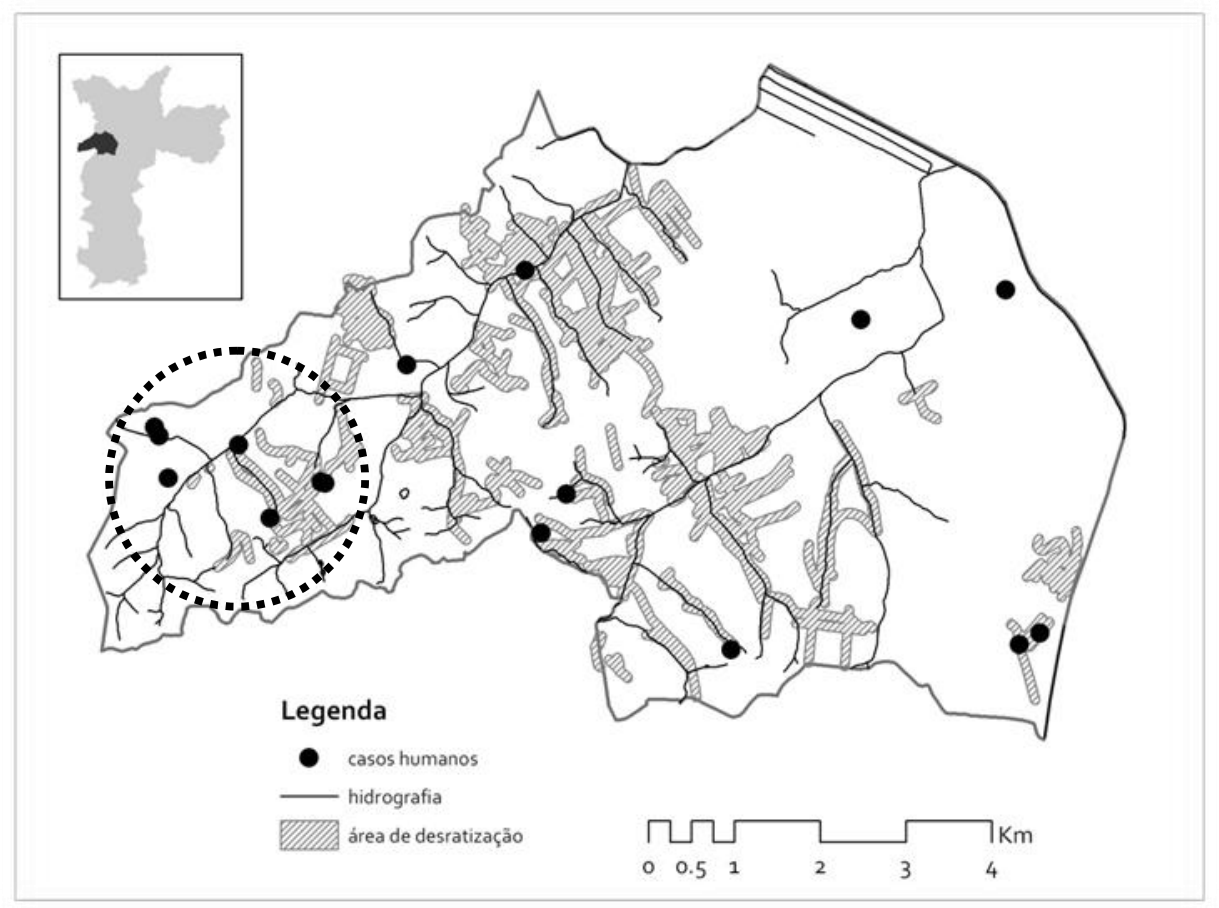

Figura 2. Localização dos casos humanos, áreas de desratização e corpos d'agua da Supervisão de Vigilância em Saúde Butantã em 2007.

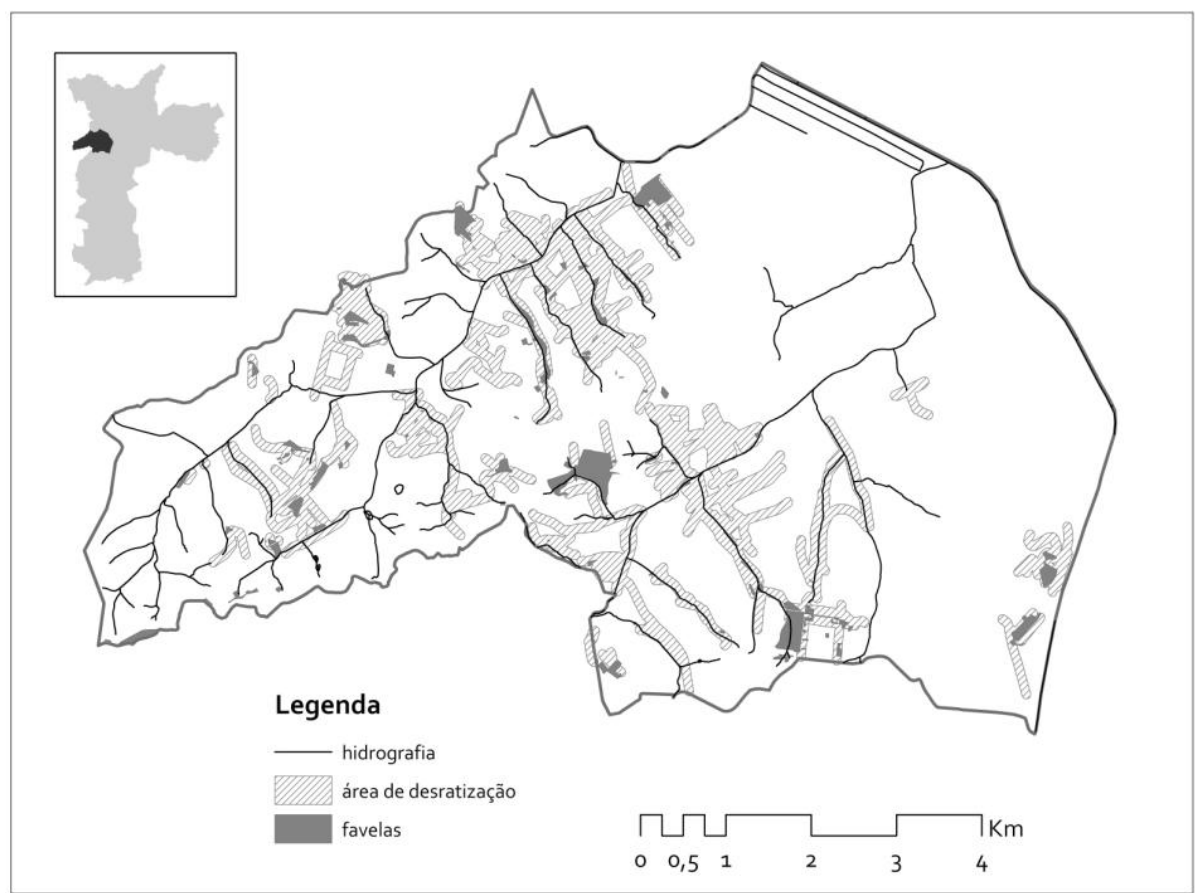

Figura 3. Localização das favelas, áreas de desratização e corpos d'água da Supervisão de Vigilância em Saúde Butantã em 2007. 
O aglomerado de focos de alagamentos foi observado ao Leste (Fig. 4), e o aglomerado de casos humanos foi observado a Oeste (Fig. 1), porém não é possível dizer que não há relação entre alagamentos e casos de leptospirose humana, uma vez que existem vastos estudos na literatura que ratificam a afinidade entre esses dois fenômenos (FUNASA, 1997). Uma justificativa para esse possível viés foi a forma de notificação dos pontos de alagamento. Essa foi feita por meio de ligações entre os observadores locais e a central do Centro de
Gerenciamento de Emergências (CGE), de modo que pontos localizados próximos a grandes vias de transporte, como avenidas e marginais, possuíam maior visibilidade e facilidade de notificação em detrimento dos pontos de alagamento situados em favelas e bairros periféricos. Nessas regiões, os alagamentos influenciam mais a ocorrência da leptospirose humana, criando condições propícias para a permanência da leptospira no ambiente (Tassinari et al., 2004).

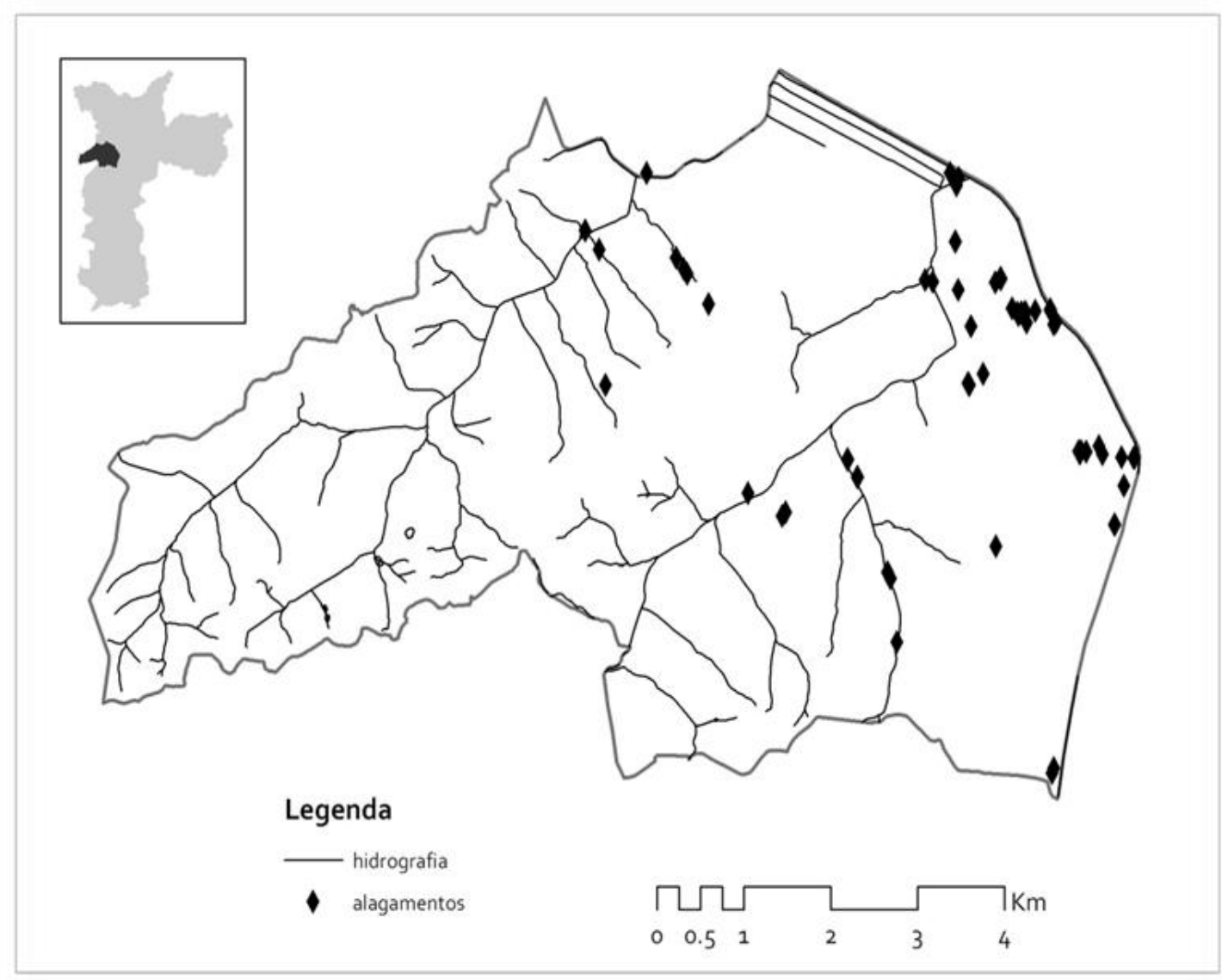

Figura 4. Localização dos pontos de alagamento em relação aos corpos d'agua na supervisão de Vigilância em Saúde Butantã em 2007.

Conforme mostra a Fig. 5, a maioria dos casos humanos $(75 \%)$ ocorreu, no máximo, 50 metros do corpo d'água. Isso reforça a ideia da importância dos ambientes inundados e úmidos sobre a sobrevivência da leptospira no ambiente e, consequentemente, a potencialidade de infecção (Lomar et al., 1996; Acha e Szyfres, 2003). 


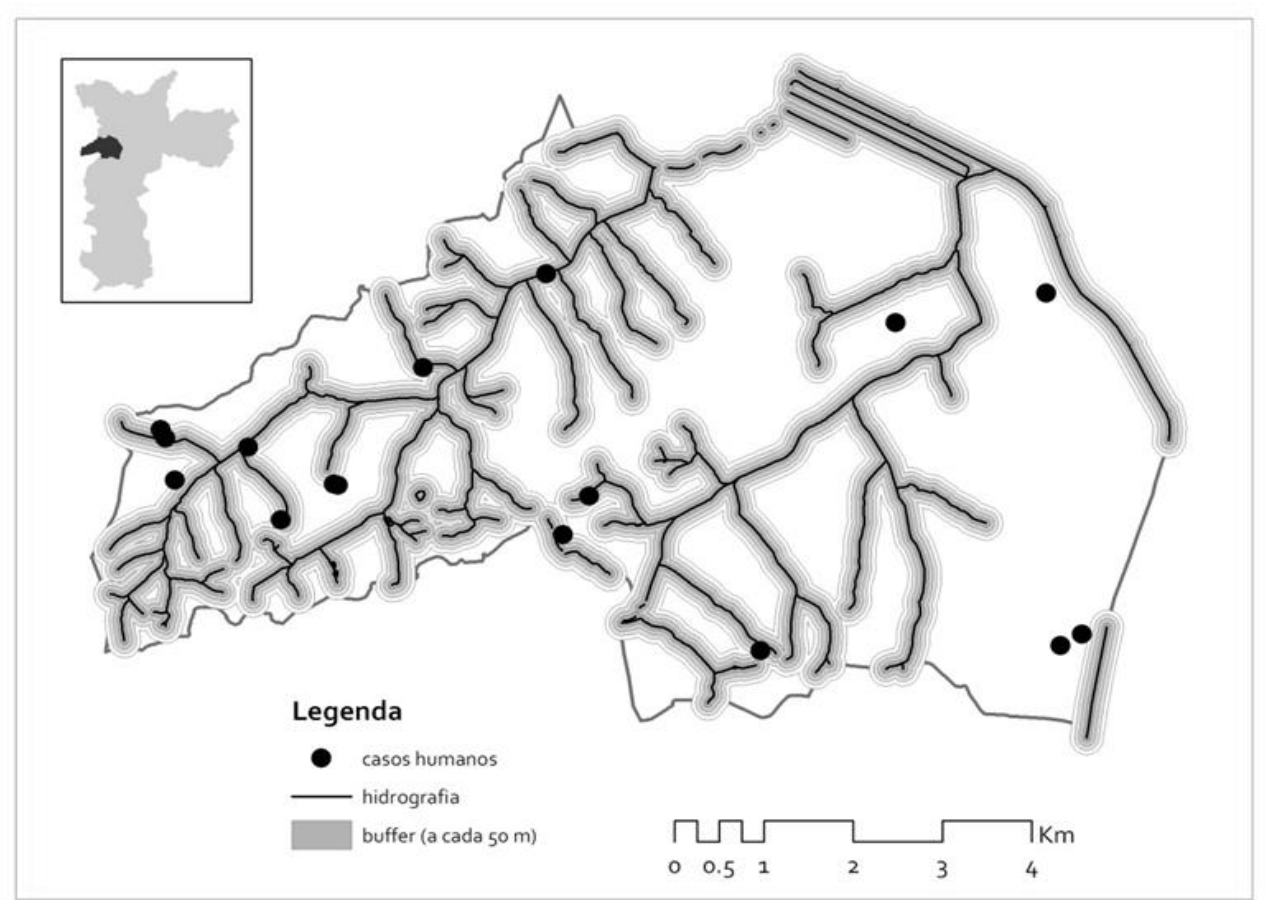

Figura 5. Localização dos casos humanos na Supervisão de Vigilância em Saúde Butantã em 2007, levando-se em conta a sua distância dos corpos d'água (os anéis mostram distâncias de 50 em 50 metros).

\section{CONCLUSÕES}

A análise da ocorrência da leptospirose humana dentro e fora das áreas de desratização mostra a relevância do programa de controle de roedores, realizado pela SUVIS Butantã, em minimizar os efeitos dos fatores de risco presentes nessas áreas. As questões levantadas neste estudo sobre o papel do cão na epidemiologia da leptospirose são importantes para direcionar novas pesquisas para a elucidação do cão como transmissor da doença para os humanos no Brasil, além de mostrar a necessidade da criação de um sistema eficaz de notificação de antropozoonoses em animais de companhia.

\section{AGRADECIMENTOS}

À FAPESP, pela bolsa de iniciação científica, e ao Laboratório de Epidemiologia e Bioestatística (FMVZ-USP), pelo apoio na realização do estudo.

\section{REFERÊNCIAS}

ACHA P.N.; SZYFRES B. Zoonosis y enfermedades transmisibles comunes al hombre y a los animales., 2.ed. Washington: Organizacion Panamericana de La Salud, 2003. p.112-120.

ALVES, C.J.; DE ANDRADE, J.S.L.; VASCONCELLOS, S.A. et al. Avaliação dos níveis de aglutininas anti-leptospira em cães no município de Patos - PB, Brasil. Rev. Bras. Cienc. Vet., v.7, p.17-21, 2000.

BARCELLOS, C.; SABROZA, P.C. The place behind the case: leptospirosis risks and associated environmental conditions in a floodrelated outbreak in Rio de Janeiro. Cad. Saúde Pública., v.17, p.59-67, 2001.

BROD, C.S.; ALEIXO, J.A.G.; JOUGLARD, S.D.D. et al. Evidência do cão como reservatório da leptospirose humana: isolamento de um sorovar, caracterização molecular e utilização em inquérito sorológico. Rev. Soc. Bras. Med. Trop., v.38, p.294-300, 2005. 
DE ALMEIDA, L.P.; MARTINS, L.F.S.; BROD, C.S. et al. Levantamento soroepidemiológico de leptospirose em trabalhadores do serviço de saneamento ambiental em localidade urbana da região sul do Brasil. Rev. Saúde Pública., v.28, p.76-81, 1994.

DE PAULA, E.V. Leptospirose Humana: uma análise climato-geográfica de sua manifestação no Brasil, Paraná e Curitiba. In: SIMPÓSIO BRASILEIRO DE SENSORIAMENTO REMOTO, 12., 2005, Goiânia. Anais...Goiânia: INPE, 2005. p.2301-2308.

IBARRA, C.; ESPINOZA, C.; CORNEJO, R. Enfermedad de weil, presentación de un caso clínico. Clin. Cienc., v.1, p.25-32, 2003.

JOUGLARD, S.D.D.; BROD, C.S. Leptospirosis in dogs: prevalence and the risk factors in the rural area of Pelotas, RS. Arq. Inst. Biol., v.67, p.181-185, 2000.
KARANDE, S.; BHATT, M.; KELKAR, A. et al. An observational study to detect leptospirosis in Mumbai, India, 2000. Arch. Dis. Child., v.88, p.1070-1075, 2003.

LOMAR, A.V.; CORREA, M.O.A.; VERONESI, R. et al. Doenças infecciosas e parasitárias. 9.ed. Rio de Janeiro: Guanabara Koogan, 1996, p.987-1003.

OIE 2006. World Organization for Animal Health. Leptospirosis. Chapter 2.2.4.

SOARES, T.S.M.; LATORRE, M.R.D.O.; LAPORTA, G.Z.; BUZAR, M.R. Análise espacial e sazonal da leptospirose no município de São Paulo, SP, 1998 a 2006. Rev. Saúde Pública, v.44, p.283-91, 2010.

TASSINARI, W.S.; PELLEGRINI, D.C.P.; SABROZA, P.C. et al. Distribuição espacial da leptospirose no Município do Rio de Janeiro, Brasil, ao longo dos anos de 1996-1999. Cad. Saúde Pública., v.20, p.1721-1729, 2004. 